\title{
Brain Connectivity Related to Working Memory Performance
}

\author{
Michelle Hampson, ${ }^{1}$ Naomi R. Driesen, ${ }^{2}$ Pawel Skudlarski, ${ }^{1}$ John C. Gore, ${ }^{3}$ and R. Todd Constable ${ }^{1}$ \\ ${ }^{1}$ Department of Diagnostic Radiology and ${ }^{2}$ Department of Psychiatry, Yale School of Medicine, New Haven, Connecticut 06520-8042, and ${ }^{3}$ Institute of \\ Imaging Science and Department of Radiology and Radiological Sciences, Vanderbilt University, Nashville, Tennessee 37232-2310
}

\begin{abstract}
Several brain areas show signal decreases during many different cognitive tasks in functional imaging studies, including the posterior cingulate cortex (PCC) and a medial frontal region incorporating portions of the medial frontal gyrus and ventral anterior cingulate cortex (MFG/vACC). It has been suggested that these areas are components in a default mode network that is engaged during rest and disengaged during cognitive tasks. This study investigated the functional connectivity between the PCC and MFG/vACC during a working memory task and at rest by examining temporal correlations in magnetic resonance signal levels between the regions. The two regions were functionally connected in both conditions. In addition, performance on the working memory task was positively correlated with the strength of this functional connection not only during the working memory task, but also at rest. Thus, it appears these regions are components of a network that may facilitate or monitor cognitive performance, rather than becoming disengaged during cognitive tasks. In addition, these data raise the possibility that the individual differences in coupling strength between these two regions at rest predict differences in cognitive abilities important for this working memory task.
\end{abstract}

Key words: task-induced deactivation; default-mode; connectivity; resting connectivity; functional connectivity; connectivity-behavior correlation; cingulate; correlated variability; FMRI; human; imaging; working memory

\section{Introduction}

Meta-analyses of functional imaging studies involving a wide range of tasks have identified a set of brain areas that typically have higher signal levels at rest than during cognitive tasks including the posterior cingulate cortex (PCC) and a medial frontal region including parts of medial frontal gyrus and ventral anterior cingulate cortex (MFG/vACC) (Shulman et al., 1997; Mazoyer et al., 2001). It has been suggested that these regions may form a default mode network that is active at rest and suspended during cognitive tasks (Raichle et al., 2001). This is a reasonable hypothesis; however, there are other plausible explanations for these deactivations that have received less attention.

It is possible that decreased metabolic activity in these regions is associated with increased engagement of the regions. An inverse relationship between activation (as measured via functional imaging) and engagement appears to exist in other parts of the brain. For example, deactivation in the hippocampus (and adjacent regions) has been reported during a transverse patterning task (TPT) and during virtual navigation of a radial arm maze (RAM) (Astur and Constable, 2004; Astur et al., 2005). These task are believed to require engagement of the hippocampus, as the ability to perform TPT or to navigate RAMs is disrupted by hip-

\footnotetext{
Received May 4, 2006; revised Oct. 17, 2006; accepted Nov. 16, 2006.

This work was supported by a Young Investigator Grant from the National Alliance for Research on Schizophrenia and Depression (N.R.D.) and by National Institutes of Health Grants R01 NS051622-01A1 (R.T.C.) and R01 EB000461 (J.C.G.).

Correspondence should be addressed to Michelle Hampson, Department of Diagnostic Radiology, Yale School of Medicine, P. 0. Box 208042, TAC-N127, New Haven, CT 06520-8042. E-mail: michelle.hampson@yale.edu. DOI:10.1523/JNEUROSCI.3408-06.2006

Copyright $\odot 2006$ Society for Neuroscience $\quad$ 0270-6474/06/2613338-06\$15.00/0
}

pocampal damage (Olton et al., 1979; Reed and Squire, 1999) (but see Bussey et al., 1998). Thus, activity in the hippocampus during these tasks appears to be inversely related to engagement. One possible mechanism for such an inverse relationship is the coding of information in terms of neural synchrony rather than rate of neural firing. Along these lines, it is interesting to note that the theta rhythm (reflecting synchronized neural activity around the $4-7 \mathrm{~Hz}$ range) is associated with memory and cognitive function (Gevins et al., 1997; Tesche and Karhu, 2000) and has been related to decreased metabolism (Uecker et al., 1997).

Thus, in some circumstance, deactivation of a brain area may be related to increased (rather than decreased) engagement. Fortunately, the degree of functional connectivity between brain areas can provide us with more direct information regarding their engagement with one another. This approach is used here to investigate the basis of deactivations in the default mode network.

Strong functional connectivity between nodes in the default mode network has been found at rest, supporting the theory that they function together during rest (Grecius et al., 2003; Laufs et al., 2003; De Luca et al., 2006; Fransson, 2005). The default mode theory would also predict that engagement between these regions should diminish during cognitive tasks, because of suppression of the circuit during task execution. However, connectivity between these regions has not been evaluated previously during cognitive tasks. In addition, the relationship between connectivity within the network and task performance has not been examined, although such connectivity-behavior analyses can provide unique insight into brain function (Hampson et al., 2006). For example, if connectivity between regions in the default mode network during a cognitive task were negatively correlated with 
task performance, it would suggest that the circuit interferes with or distracts from cognitive processing.

This study tested the hypotheses that connectivity between the MFG/vACC and PCC differs during a working memory task and rest, and that connectivity between the two regions is related to task performance. Our hypotheses [on which our region-ofinterest (ROI) analyses are based] focus on these two regions because they are frequently deactivated during functional imaging studies (Shulman et al., 1997; Mazoyer et al., 2001) and because their resting connectivity patterns in healthy adults have been described previously (Grecius et al., 2003).

Interregional connectivity was assessed via two different methods. First, a seed region approach was used. This approach had the advantage that it provided connectivity maps for visualization, but the disadvantage that regions were not defined in the same manner, because the seed was treated in a special way. Therefore, a second analysis that defined the two regions in an identical manner was also performed (as suggested by an anonymous reviewer of this paper).

\section{Materials and Methods Subjects}

Data from nine healthy subjects (ages $28-45$; five women and four men) were analyzed. Data collection and analyses were approved by the $\mathrm{Hu}-$ man Investigations Committee of the Yale School of Medicine (New Haven, CT).

\section{Data acquisition}

Subjects were scanned in a GE (Waukesha, WI) 1.5T Signa LX scanner. T1-weighted anatomical data were collected for $165 \mathrm{~mm}$ axial slices (parallel to a line passing through the anterior and posterior commissures). Twelve functional imaging scans were then collected in the same locations, using a $\mathrm{T} 2^{\star}$-sensitive gradient-recalled, single-shot echoplanar pulse sequence (repetition time, $1500 \mathrm{~ms}$; echo time, $50 \mathrm{~ms}$; flip angle, $70^{\circ}$; $64 \times 64$ matrix; $20 \times 20 \mathrm{~cm}$ field of view). Each scan was 3 $\mathrm{min}, 12 \mathrm{~s}$ long and involved the collection of 128 images (first four were discarded).

\section{Experimental paradigm}

Of the 12 functional scans, two were block design and alternated three times between baseline and verbal working memory blocks. There was a $1 \mathrm{~s}$ introduction to each block followed by the presentation of 10 stimuli (each presented for $500 \mathrm{~ms}$ followed by $2500 \mathrm{~ms}$ of a checkerboard mask) for a total of 31 s per block. In the baseline task, subjects pressed one button each time the letter A appeared on the screen and another button when other letters appeared. The remaining 10 scans were steady state scans used for functional connectivity analyses. Five of these were resting scans. In the other five, subjects performed the three-back task throughout the scan. Scan types were interleaved in a manner that varied across subjects.

\section{Data analyses \\ Preprocessing}

Data were motion corrected using the SPM99 (Statistical Parametric Mapping 99) algorithm (http://www.fil.ion.ucl.ac.uk/spm/). A spatial Gaussian filter (two pixels full-width at half maximum) was applied. Pixels with a median value below one-fifth of the maximum median pixel value were set to zero. For the data used in correlational analyses, both a high-pass filter $(0.01 \mathrm{~Hz})$ and a low-pass filter $(0.2 \mathrm{~Hz})$ were applied. The cutoff for the low-pass filter was selected to preserve the blood oxygen level-dependent signal while removing higher-frequency noise.

\section{Activation analyses (block design paradigm)}

Images from the two block-design scans were assigned to task or baseline blocks after adjusting for hemodynamic delay. For each subject at each pixel, percent signal change across conditions was computed in each of the two scans separately and averaged across the scans, yielding a map representing that subject's percent signal change in the block-design scans. These maps were transformed to Talairach space and the percent signal changes with conditions for each pixel were compared across subjects (using a two-tailed $t$ test) to a distribution with a mean of zero to yield a composite map of the significance of activations/deactivations to the block-design task.

For each subject, a $t$ test was also performed at each pixel comparing signal level during task to signal level during baseline in each scan and averaged across scans to yield a t-map of activation for that subject. These t-maps were used to functionally define the seed region (MFG/vACC) to be used in a seed-region based correlation analysis. The MFG/vACC seed region was defined in each subject to be that subject's eight most deactivated pixels falling within the MFG [Brodmann's area (BA) 9/10] or the $\operatorname{vACC}($ BA24/32 below $z=26$ ). The total volume from which these pixels were selected encompasses $\sim 19 \mathrm{~cm}^{3}$ in the Talairach brain.

\section{Functional connectivity maps}

The reference time course for a given subject was computed by averaging the time course across all eight pixels in their functionally defined MFG/ vACC reference region. That reference time course was then correlated with the time course of every other pixel in the brain within each resting or continuous working memory scan, after removing the effect of the mean time course of the slice in which the pixel was located.

For each of the steady-state scans, the resulting correlations were transformed to a Gaussian distribution via Fisher's transformation. By fitting the distribution (to full-width at half maximum) with a Gaussian and adjusting for SD and mean, the data from each map were transformed to a standard normal distribution (Lowe et al., 1998; Hampson et al., 2002). The maps from the resting scans of each subject were then averaged (and adjusted for the decrease in SD associated with that averaging) to yield a map representing the strength of resting state functional connectivity to the MFG/vACC area in terms of standard normal Gaussian variables ( $z$ values). Similarly, the $z$-transformed correlation maps were combined across continuous working memory scans to yield a map representing functional connectivity to the MFG/vACC during the working memory scans in terms of $z$ values.

\section{Composite functional connectivity maps}

The resting functional connectivity map of each subject was transformed to Talairach space, and a pixel-wise $t$ test was applied to produce a map of the significance of resting correlation to the MFG/vACC across subjects. Similarly, a composite map of correlations to the MFG/vACC in the working memory condition was computed.

\section{Assessing strength of functional connectivity in each subject}

Method 1: seed region-based analysis (based on MFG/vACC correlation maps). The composite activation map was thresholded at $p=0.005$ and cluster filtered to remove activations of $<10$ pixels. The PCC ROI was defined to include all deactivated pixels in the PCC (BA23, BA31) and contiguous deactivation extending into the precuneus (BA7). For each condition, in each subject, strength of the MFG/vACC to PCC correlation was assessed as the average $z$-transformed correlation to MFG/vACC across all pixels in the PCC ROI of the Talairach-transformed correlation map for that subject.

Method 2: direct correlation of average ROI time courses. The MFG/ vACC and PCC ROIs were both defined based on the composite activation map and were propagated back to the individual subjects' brains (using an inverse Talairach transform). For each scan of each subject, the average time courses of the two ROIs were correlated and the resulting $r$ values transformed to a Gaussian distribution (with an expected mean of zero because of slice mean removal) via Fischer's transform. These Gaussian values were averaged across resting scans to yield an estimate of resting state functional connectivity, and across working memory scans to yield an estimate of connectivity in the working memory condition.

\section{Significance of connectivity strength in different conditions}

$t$ tests were used to assess the significance of functional connectivity across subjects in each condition, and the change in connectivity with condition. 
Connectivity-behavior correlations

Working memory performance for each subject was assessed as the average percent correct across continuous working memory scans. These numbers were correlated across subjects with the strength of subjects' MFG/vACC to PCC correlations (as assessed via each of the two methods) in the resting and working memory conditions separately, and transformed to $p$ values via a standard $r$-to- $z$ transform $(n=9)$. The change in connectivity across conditions was also correlated with working memory performance.

\section{Results}

\section{Activations and deactivations to} working memory task

The working memory task activated the dorsolateral prefrontal cortex bilaterally (BA9/46), left ventrolateral prefrontal cortex (BA44), left premotor cortex (BA6), right frontal pole (BA 10), bilateral inferior parietal lobule (BA40), a region at the junction of dorsal anterior cingulate cortex and medial frontal gyrus (BA8/32), right insula, right temporal gyrus (BA21/ 37 ), and a subcortical region at the junction of the left thalamus, caudate, and lenticular nucleus. These areas are highly consistent with activations reported in other working memory studies that have used a verbal $n$-back task (Owen et al., 2005). More importantly, the task deactivated a typical example of the default mode network, including the MFG/vACC, PCC, and portions of left BA39/ 19. The composite activation map is shown in Figure 1.

\section{Connectivity analyses}

Because the two methods of assessing functional connectivity strength yielded the same findings for both the ROI analyses and the connectivity-behavior correlations, only those from Method 1 are presented.

Similar to the findings reported by Grecius (2003), the region showing the most pronounced positive correlation to the MFG/ vACC during rest, aside from itself, was the posterior cingulate cortex (Fig. 2a). ROI analysis revealed a significant correlation between the MFG/vACC and PCC at rest $(p=0.003)$.

Bilateral loci of positive correlation to the MFG/vACC were also found in the middle and superior frontal gyri (BA8/9) and in the inferior parietal cortex (BA39/40). Although these were not identified in the vACC functional connectivity analysis of the Grecius (2003) study, they are proximal to loci reported in studies of resting functional connectivity to PCC (Grecius et al., 2003; Fransson, 2005) and are regions that were identified in the original meta-analyses of areas that show decreased signal to a variety of tasks (Shulman et al., 1997; Mazoyer et al., 2001). Thus, the areas of positive connectivity to the MFG/vACC at rest (Fig. 2a) are consistent with our knowledge of the default mode circuit.

During the working memory task, the posterior cingulate cortex was still apparent in the MFG/vACC composite correlation map (Fig. 2b), and ROI analysis once again revealed a significant correlation between the two regions during the working memory task $(p=0.004)$. Although the strength of correlation to the MFG/vACC in the PCC appears diminished in the working memory condition relative to the resting condition in the com- posite correlation maps, ROI analysis did not reveal a significant difference across condition (possibly because of power limitations).

Other loci showing positive correlations to the MFG/vACC during the working memory task include left superior frontal gyrus (BA9), left sensorimotor cortex (BA3), cuneus (BA19), rostral PCC (BA31/24), and two loci in the left middle temporal gyrus (BA39 and BA21). Many of these areas have been associated with the default mode circuit in previous studies. More specifically, the left superior frontal gyrus was identified in the first meta-analyses of regions deactivating to a range of tasks (Shulman et al., 1997), as well as in studies of resting connectivity to PCC (Grecius et al., 2003; Fransson, 2005). Both loci in the middle temporal gyrus are proximal to regions that were reported to be functionally connected to the PCC at rest, and the rostral PCC region has been reported previously to be functionally connected with vACC at rest (Grecius et al., 2003). Thus, during task, as well as at rest, MFG/vACC is functionally connected with many areas that have been previously implicated in the default mode network.

It is interesting to note that several classic working memory areas can been seen as negative correlations in the maps of connectivity with the MFG/vACC, and particularly in the map of connectivity during the working memory task (Fig. 2b). These include the bilateral dorsolateral prefrontal cortex, left ventrolateral prefrontal cortex, and the inferior parietal lobule.

\section{Connectivity-behavior correlations}

The connectivity-behavior analysis revealed that working memory performance was significantly positively correlated with MFG/vACC to PCC connectivity in the resting condition ( $p=$ 0.01 ). Working memory performance was also significantly positively correlated with MFG/vACC to PCC connectivity in the working memory condition $(p=0.04)$. Working memory per- 


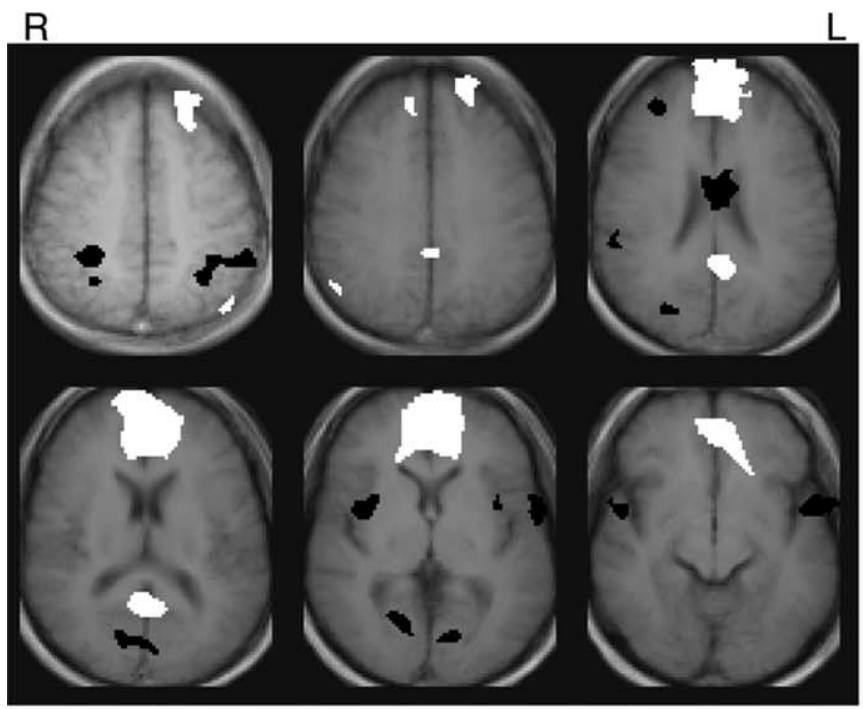

(a)

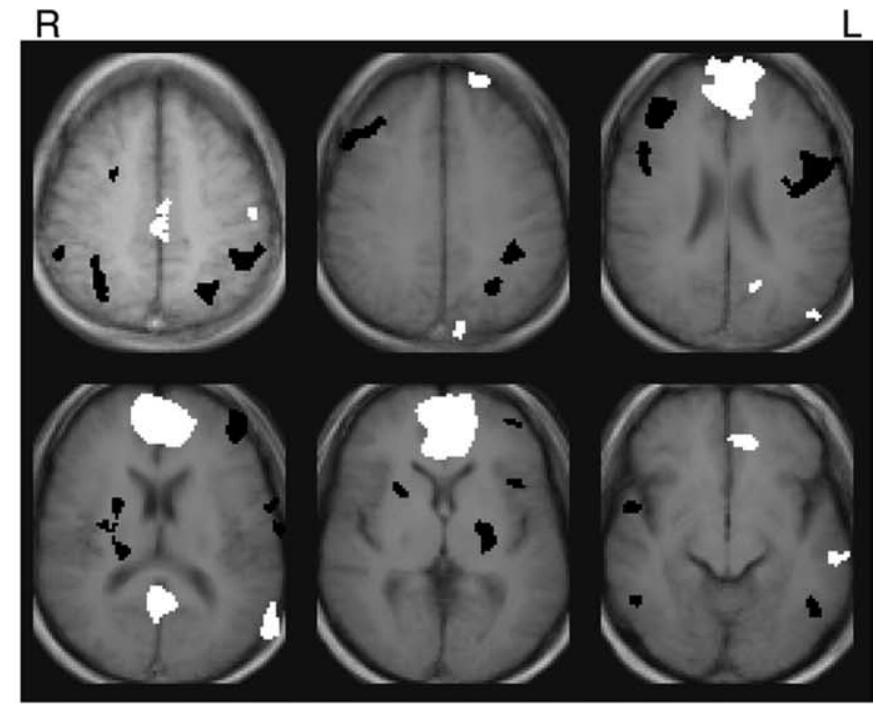

(b)

Figure 2. $\boldsymbol{a}, \boldsymbol{b}$, Composite maps of correlations to MFG/vACC during resting scans ( $\boldsymbol{a})$ and working memory scans $(\boldsymbol{b})(p<0.005$, uncorrected). Positive correlations are white and negative correlations are black.

Table 1. Connectivity and performance data

\begin{tabular}{|c|c|c|c|c|c|c|}
\hline \multirow[b]{2}{*}{ Subject number } & \multicolumn{2}{|c|}{$\begin{array}{l}\text { Strength of functional } \\
\text { connection }\end{array}$} & \multicolumn{4}{|c|}{ Performance during task (\%) } \\
\hline & Rest & Task & Correct & Misses & False hits & Invalid responses* \\
\hline 1 & 1.03 & 1.40 & 79.3 & 17.9 & 2.8 & 0 \\
\hline 2 & 3.63 & 3.43 & 90.0 & 7.7 & 1.3 & 1 \\
\hline 3 & 3.28 & 2.85 & 92.7 & 4.3 & 3.0 & 0 \\
\hline 4 & 1.51 & 1.75 & 81.9 & 10.4 & 6.0 & 1.7 \\
\hline 5 & 0.70 & 0.91 & 83.3 & 10.3 & 1.0 & 5.3 \\
\hline 6 & 7.19 & 8.86 & 97.0 & 2.0 & 1.0 & 0 \\
\hline 7 & 7.59 & 4.23 & 95.0 & 4.4 & 0.7 & 0 \\
\hline 8 & 2.15 & 1.31 & 94.6 & 3.3 & 2.1 & 0 \\
\hline 9 & 4.67 & 5.06 & 98.3 & 1.7 & 0 & 0 \\
\hline
\end{tabular}

Connectivity strength is a Gaussian variable (obtained as described in Materials and Methods). Performance is presented in percentages. *Invalid responses occurred when subjects did not respond to stimuli or gave both a "yes" and a "no" response to the same stimulus.

formance was not significantly correlated with changes in connectivity across conditions. Table 1 provides data on individual subjects' functional connectivity and their performance. It is interesting to note that the pattern of connectivity strength across subjects (Table 1, columns 1,2) was highly correlated for the two conditions $(p=0.002)$.

\section{Discussion}

\section{Physiologic noise}

Physiological noise in functional connectivity studies can reduce power or yield artifactual correlations (Lowe et al., 1998; Raj et al., 2001; Birn et al., 2006; Lund et al., 2006). These noise sources include cardiac-induced signal changes (Dagli et al., 1999), artifacts arising in synchrony with the respiratory cycle (Raj et al., 2001), and low-frequency variations in respiration rate or depth (Birn et al., 2006). The last of these noise sources is particularly problematic for this study because the spatial location of artifacts related to these respiratory effects was found to overlap with the default mode network (Birn et al., 2006).

The time courses of physiological fluctuations were not recorded in this study and, thus, could not be retrospectively removed from the data. However, a recent study reported that regressing out global signal change provided comparable results to the recording and subsequent removal of low-frequency respi- ratory fluctuations (Birn et al., 2006). In this study, a similar approach was adopted, with the modification that the mean time course of the slice was removed rather than the global time course. Because it is done on a slice level, this approach is better at reducing noise caused by transient physiologic fluctuations that occur during part of the volume collection and that can lead to elevated correlations between slices collected next to one another in time.

We expect the removal of slice mean to be most successful for respiratory noise, because the motion and susceptibility effects associated with respiration are relatively broadly spatially distributed. Cardiac effects are more localized and, thus, less well characterized in the mean signal; however, examination of the correlation maps does not reveal patterns similar to those shown (Dagli et al., 1999) to characterize cardiac noise. Therefore, in our opinion, it is unlikely that our findings are attributable to such confounds. Future studies of functional connectivity, and particularly those involving the default mode network (as it includes areas where there is substantial physiologic noise), could address this issue more thoroughly by collecting data on physiological fluctuations during the scans. A variety of methods to remove noise arising from monitored physiological sources that are under-sampled in the imaging data have been described previously (Hu et al., 1995; Glover et al., 2000; Lund et al., 2006).

\section{Implications for default mode theory}

A significant correlation was found between the MFG/vACC and PCC in the resting state. This is consistent with previous studies reporting that these regions are components in a network of regions that show coherent signal fluctuations at rest (Grecius et al., 2003; Laufs et al., 2003; De Luca et al., 2006; Fransson, 2005), and supports the possibility that these regions function together during rest, as suggested in the default mode hypothesis. 
A significant correlation was also found between the MFG/ vACC and PCC during the working memory task, suggesting that the network was still engaged during the task. This finding can be explained in terms of the default mode hypothesis by assuming that subjects were not continuously performing the task and, thus, were intermittently activating the default mode network, and/or by positing that default mode processes can be carried on in parallel with task performance. People commonly experience mental events that are unrelated to immediate sensory input. This phenomenon, referred to as stimulus-independent mentation, or SIM (Singer, 1988), may be an aspect of default mode processing. All subjects in this study were scoring $>75 \%$ on a challenging (three-back) working memory task, suggesting that they were attending well to the task. However, SIM has been found to occur even during cognitive tasks that require attention, such as a pitch-comparison task with stimuli arriving once per second (Antrobus et al., 1966). Thus, it is possible subjects in this study were engaging in SIM during their working memory scans and that connectivity between the MFG/vACC and PCC in the task condition is related to this task-irrelevant processing.

However, the positive correlation between task performance and MFG/vACC to PCC connectivity during the working memory scans was surprising in this context. If task-irrelevant mental processes compete with task processes for cognitive resources, we would expect a negative relationship between task performance and the degree of engagement of the default mode circuit. For example, in a previous study, occurrence of SIM was associated with decreased cognitive performance (Teasdale et al., 1995). Similarly, in this study, a negative correlation between working memory performance and task-irrelevant mental processing (and thus MFG/vACC to PCC connectivity) would be expected. On the contrary, a significant positive correlation between performance and connectivity was found. Thus, it does not appear that engagement between these two regions detracts from cognitive performance.

\section{Metabolism, interregional connectivity, and performance}

Recent studies have reported regional changes in activity that are inversely related to performance (Otten and Rugg, 2001; Daselaar et al., 2004; Drummond et al., 2005; Gonsalves et al., 2005; Polli et al., 2005). These studies highlight the importance of examining the role of deactivations as well as activations when studying brain function. However, studies such as these that examine the relationship between regional metabolic activity level and performance cannot address how the engagement of regions within a given network is related to performance, because it is unknown how deactivations are related to inter-regional interaction. It is possible that a region may deactivate when the circuit it belongs to is suppressed and the decreased interaction between component regions results in decreased metabolic demand. However, it is also theoretically possible that a region's firing rate could drop below baseline when its neural firing patterns become phaselocked with other regions if that phase-locking involves a lower firing rate than baseline. In the first case, deactivation would be associated with decreased engagement between regions, in the latter, it would be associated with increased engagement between regions. In this study, a complementary approach is used that is designed to examine specifically how the engagement between regions is related to cognitive performance. By correlating behavior with inter-regional functional connectivity (rather than with regional activation measures), the inter-regional engagement is related directly to performance.

This approach has revealed the surprising finding that work- ing memory performance is positively related to functional connectivity in the so-called default mode network (as measured both at rest and during task performance). This suggests that these areas may be part of a network that facilitates cognitive performance. Given that the resting scans were interleaved with the working memory scans, it is also possible that the strength of this connection was a reflection of performance, rather than a predictor of it. Finally, we cannot rule out the possibility that the correlation is incidental and that the interaction between these regions does not directly influence or reflect performance (e.g., perhaps some third variable influences both connectivity and performance). Regardless of the precise basis of this finding, the positive relationship between functional connectivity and performance challenges the view that interactions between component regions of the default mode network detract from cognitive performance.

More generally, correlations between brain connectivity and behavior provide a powerful tool for investigating individual differences and their relationships to neurocircuitry (Hampson et al., 2006). For example, in a previous study of effective connectivity between brain areas during a working memory task, correlations were computed between effective connectivity and both performance and self-reported strategy measures. These correlations yielded insights into the brain networks underlying effective (and less effective) working memory strategies (Glabus et al., 2003). In the current study, a pattern of connectivity across subjects was found that was similar during rest and task scans, and that was related to performance. The stability of the pattern across conditions suggests that the strength of this brain connection may be related to some stable subject trait such as a cognitive skill or a personality dimension, which in turn may be related to performance. Many studies have examined the relationship between personality and brain activity, as measured via a variety of functional imaging techniques (Ebmeier et al., 1994; Fischer et al., 1997; Canli et al., 2001; Gusnard et al., 2003). It would be interesting to extend this approach by examining the relationship between personality and brain connectivity, and in particular, to see how connectivity between the MFG/vACC and PCC is related to personality dimensions.

\section{Clinical implications}

Interestingly, a previous study found weaker connectivity in the "default mode" network of subjects with Alzheimer's disease relative to healthy older subjects (Grecius et al., 2004). Because episodic memory has been proposed as one of the possible resting state activities that the default mode circuit engages in, the findings in that study were interpreted in terms of disrupted episodic memory function in Alzheimer's disease. However, decreased connectivity within the so-called default mode network of Alzheimer's patients is also consistent with our findings that connectivity in the circuit is related to performance on demanding cognitive tasks, as Alzheimer's disease is associated with a general disruption in cognitive function.

More generally, if resting state connectivity between these regions is a marker of particular cognitive abilities, the strength of this connection could provide diagnostic information across a range of mental disorders that involve disrupted cognitive function including schizophrenia, Alzheimer's disease, and mild cognitive impairment. The pattern of connectivity between these regions, and also the patterns of their connectivity to other parts of the brain, should be examined and related to clinical conditions. 


\section{References}

Antrobus JS, Singer JL, Greenberg S (1966) Studies in the stream of consciousness: experimental enhancement and suppression of spontaneous cognitive processes. Percept Mot Skills 23:399-417.

Astur RS, Constable RT (2004) Hippocampal dampening during a relational memory task. Behav Neurosci 118:667-675.

Astur RS, St. Germain SA, Baker EK, Calhoun V, Pearlson GD, Constable RT (2005) FMRI hippocampal activity during a virtual radial arm maze. Appl Psychophysiol Biofeedback 30:307-317.

Birn RM, Diamond JB, Smith MA, Bandettini PA (2006) Separating respiratory-variation-related fluctuations from neuronal-activity-related fluctuations in fMRI. NeuroImage 31:1536-1548.

Bussey TJ, Warburton EC, Aggleton JP, Muir JL (1998) Fornix lesions can facilitate acquisition of the transverse patterning task: a challenge for "configural" theories of hippocampal function. J Neurosci 18:1622-1631.

Canli T, Zhao Z, Desmond JE, Kang E, Gross J, Gabrieli JD (2001) An fMRI study of personality influences on brain reactivity to emotional stimuli. Behav Neurosci 115:33-42.

Dagli MS, Ingleholm JE, Haxby JV (1999) Localization of cardiac-induced signal change in fMRI. NeuroImage 9:407-415.

Daselaar SM, Prince SE, Cabeza R (2004) When less means more: deactivations during encoding that predict subsequent memory. NeuroImage 23:921-927.

De Luca M, Beckman CF, De Stefano N, Matthews PM, Smith SM (2006) FMRI resting state networks define distinct modes of long-distance interactions in the human brain. NeuroImage 29:1359-1367.

Drummond SP, Bischoff-Grethe A, Dinges DF, Ayalon L, Mednick DC, Meloy MJ (2005) The neural basis of the psychomotor vigilance task. Sleep 28:1059-1068.

Ebmeier KP, Deary IJ, O'Carroll RE, Prentice N, Moffoot APR, Goodwin GM (1994) Personality associations with the uptake of the cerebral blood flow marker 99mTc-exametazime estimated with single photon emission tomography. Pers Individ Dif 17:587-595.

Fischer H, Wik G, Fredrikson M (1997) Extraversion, neuroticism and brain function: a PET study of personality. Pers Individ Dif 23:345-352.

Fransson P (2005) Spontaneous low-frequency BOLD signal fluctuations: an $\mathrm{fMRI}$ investigation of the resting-state default mode of brain function hypothesis. Hum Brain Mapp 26:15-29.

Gevins A, Smith ME, McEvoy L, Yu D (1997) High-resolution EEG mapping of cortical activation related to working memory: effects of task difficulty, type of processing, and practice. Cereb Cortex 7:374-385.

Glabus MF, Horwitz B, Holt JL, Kohn PD, Gerton BK, Callicott JH, MeyerLindenberg A, Berman KF (2003) Interindividual differences in functional interactions among prefrontal, parietal and parahippocampal regions during working memory. Cereb Cortex 13:1352-1361.

Glover GH, Li T-Q, Ress D (2000) Image-based method for retrospective correction of physiological motion effects in fMRI: RETROICOR. Magn Res Med 44:162-167.

Gonsalves BD, Kahn I, Curran T, Norman KA, Wagner AD (2005) Memory strength and repetition suppression: multimodal imaging of medial temporal cortical contributions to recognition. Neuron 47:751-761.

Grecius MD, Krasnow B, Reiss AL, Menon V (2003) Functional connectivity in the resting brain: A network analysis of the default mode hypothesis. Proc Natl Acad Sci USA 100:253-258.

Grecius MD, Srivastava G, Reiss AL, Menon V (2004) Default-mode network activity distinguishes Alzheimer's disease from healthy aging: evidence from functional MRI. Proc Natl Acad Sci USA 101:4637-4642.

Gusnard DA, Ollinger JM, Shulman GL, Cloninger CR, Price JL, Essen DCV,
Raichle ME (2003) Persistence and brain circuitry. Proc Natl Acad Sci USA 100:3479-3484.

Hampson M, Peterson BS, Skudlarski P, Gatenby JC, Gore JC (2002) Detection of functional connectivity using temporal correlations in MR images. Hum Brain Mapp 15:247-262.

Hampson M, Tokoglu F, Sun Z, Schafer RJ, Skudlarski P, Gore JC, Constable RT (2006) Connectivity-behavior analysis reveals that functional connectivity between left BA39 and Broca's area varies with reading ability. NeuroImage 31:513-519.

Hu X, Le TH, Parrish T, Erhard P (1995) Retrospective estimation and correction of physiological fluctuation in functional MRI. Magn Res Med 34:201-212.

Laufs H, Krakow K, Sterzer P, Eger E, Beyerle A, Salek-Haddadi A, Kleinschmidt A (2003) Electroencephalographic signatures of attentional and cognitive default modes in spontaneous brain activity fluctuations at rest. Proc Natl Acad Sci USA 100:11053-11058.

Lowe MJ, Mock BJ, Sorenson JA (1998) Functional connectivity in single and multislice echoplanar imaging using resting-state fluctuations. NeuroImage 7:119-132.

Lund TE, Madsen KH, Sidaros K, Luo WL, Nichols TE (2006) Non-white noise in fMRI: does modelling have an impact? NeuroImage 29:54-66.

Mazoyer B, Zago L, Mellet E, Bricogne S, Etard O, Houdé O, Crivello F, Joliot M, Petit L, Tzourio-Mazoyer N (2001) Cortical networks for working memory and executive functions sustain the conscious resting state in man. Brain Res Bull 54:287-298.

Olton DS, Becker JT, Handelmann GE (1979) Hippocampus, space and memory. Behav Brain Sci 2:313-365.

Otten LJ, Rugg MD (2001) When more means less: neural activity related to unsuccessful memory encoding. Curr Biol 11:1528-1530.

Owen AM, McMillan KM, Laird AR, Bullmore E (2005) N-back working memory paradigm: a meta-analysis of normative functional neuroimaging studies. Hum Brain Mapp 25:46-59.

Polli FE, Barton JJ, Cain MS, Thakkar KN, Rauch SL, Manoach DS (2005) Rostral and dorsal anterior cingulate cortex make dissociable contributions during antisaccade error commission. Proc Natl Acad Sci USA 102:15700-15705.

Raichle ME, MacLeod AM, Snyder AZ, Powers WJ, Gusnard DA, Shulman GL (2001) A default mode of brain function. Proc Natl Acad Sci USA 98:676-682.

Raj D, Anderson AW, Gore JC (2001) Respiratory effects in human functional magnetic resonance imaging due to bulk susceptibility changes. Phys Med Biol 46:3331-3340.

Reed JM, Squire LR (1999) Impaired transverse patterning in human amnesia is a special case of impaired memory for two-choice discrimination tasks. Behav Neurosci 113:3-9.

Shulman GL, Fiez JA, Corbetta M, Buckner RL, Miezin FM, Raichle ME, Petersen SE (1997) Common blood flow changes across visual tasks: II. Decreases in cerebral cortex. J Cogn Neurosci 9:648-663.

Singer JL (1988) Sampling ongoing consciousness and emotional experience: implications for health. In: Psychodynamics and cognition (Horowitz MJ, ed), pp 297-346. Chicago: Chicago UP.

Teasdale JD, Dritschel BH, Taylor MJ, Proctor L, Lloyd CA, Nimmo-Smith I, Baddeley AD (1995) Stimulus-independent thought depends on central executive resources. Mem Cognit 23:551-559.

Tesche CD, Karhu J (2000) Theta oscillations index human hippocampal activation during a working memory task. Proc Natl Acad Sci USA 97:919-924.

Uecker A, Barnes CA, McNaughton BL, Reiman EM (1997) Hippocampal glycogen metabolism, EEG, and behavior. Behav Neurosci 111:283-291. 